\section{sciendo}

Management Consulting Journal

Volume 5.1 | January 2022

DOI: $10.2478 / \mathrm{mcj}-2022-0005$

ISSN: 2631-987X

() 2022 Ton Berendsen. This is an open access article licensed under the Creative Commons Attribution-NonCommercial-NoDerivs License (http://creativecommons.org/licenses/by-nc-nd/3.0/).

\title{
The Role of Psychology in Consulting - Some Considerations
}

\author{
Ton Berendsen
}

\section{Introduction}

My ambition with this contribution is to provide some deliberations regarding the possible functions of psychology in management consultancy. From my studies of psychology to Masters level and experience as a consultant, a number of analogies will be explored between consultancy and psychology. The value thereof is that these disciplines hold different assumptions and views as well as raise another kind of questions, which may be mutually beneficial. Furthermore, consultants may already use achievements, derived from the psychology. At the end of this contribution, some questions for further debate will be summarised.

\section{Psychology and Consulting}

Positioning. Both consultancy and psychology may be perceived as an intersubjective combination of diagnosing and constructing, followed by changing a less desired diagnosed state into a more desired constructed state. In consultancy, one of the many distinctions is between "hard" (for instance structures and systems) and "soft" (for instance behaviours and competences) phenomena. Although this distinction can be made in theory, it certainly cannot always be made in practice, among others given the strong interdependencies as well as the overlaps between the "hard" and the "soft". A similar line of reasoning may be followed in relation to psychology, where a stricter, but nevertheless still artificial distinction, is made between the mind (the more "soft" side, theoretically the specialism of psychology) and the brain (the more "hard" side, theoretically the specialism of psychiatry or neurology). Following this, the core of consultancy and psychology is changing a less desired state into a more desired state, which should cover both "hard" and "soft" phenomena.

Process. Consultancy and psychology often follow sequential processes. A first subprocess is diagnosing, focused on tracking down the problems or complaints and their trigger events, as well as the underlying causes and the maintaining processes. A second subprocess is constructing, directed at creating a model for improved functioning, taking into account the diagnosis undertaken plus the feasible options. A third subprocess is changing, thus advancing the diagnosed less desired state into the 
constructed more desired state. The term "more desired state" also implies solving or at least softening the problems or complaints and their consequences as well as taking away or at least mitigating the underlying maintaining processes as much as possible. This of course in order to prevent the reoccurrence of more or less the same problems or complaints in organisations or persons.

Reality. Consultants and psychologists have to create models of "the reality", among which diagnostic models of the current state and constructed models of the desired state. This modelling is an art, and distortions may occur. This starts with the notion that reality is a social construct, implying that many "realities" coexist, among others stemming from different backgrounds and paradigms as well as differences in mindsets and beliefs. Also, in the interactions of consultants or psychologists with their clients, information has to be derived and opinions have to be formed, all subject to many filters and biases. Likewise, legitimations and confabulations from clients may be presented to protect own contributions.

Detailing. Furthermore, in consultancy and psychology all kinds of different decompositions are applied. Broader dividing or deeper detailing of situations may be productive only if and when this will provide a better understanding of the situation and foremost will contribute to a better quality of the solution. In cases of a too deep detailing, it may well be possible that track of the overarching is lost. This complexity in diagnoses may also spill over to the design and even the change itself, hampering them. All of this may possibly be further aggravated by overspecialisation, which may give rise to the fixation on a limited set of aspects of problems or the promotion of a "best" solution. In extreme cases, overspecialisation may even lead to antagonism between professionals, induced by tribal struggles. That being said, in terms of "breadth", it is of the utmost importancy, that a total ecosystem is in scope in the whole process.

Emphasis. In realising a more desired state, another continuum should be taken into account, ranging from a more exclusive focus on the "product" to a more exclusive focus on the "process". Consultants and psychologists make an informed choice around the situational emphasis in bridging the distance between the diagnoses state and the constructed state of organisations. Given that organisations are composed of individuals, this implies that organisational change also includes adapting or changing the behavior of leaders and other persons, as it is the case in psychology. Of course, apart from these similarities, there are also many differences between consultancy and psychology, but these are considered as less relevant from the point of view of this contribution.

Normality. In consultancy and psychology, nearly all phenomena in organisations and persons may form normal distributions. This implies that all positions of a certain distribution are variants of, more or less, the "normal". Another more fundamental judgement is determining to what extent problems or complaints may be classified as normal or abnormal and from what point intervention is really required instead of desired. There can be no clear distinction between normal versus abnormal in consultancy nor in psychology. Apart from the subjectivities and other difficulties in appreciating phenomena, this is for instance because behavior of persons should be appreciated situationally. This implies that certain behaviors may be highly productive in 
one context or situation and may be strongly counterproductive in another. However, in consultancy as in psychology, there is an indication that immediate intervention is inescapable. This indicator is causing danger to oneself (for instance, in organisations continuity pressure and in persons suicide ideation) or to others (for instance, in organisations pollution issues and in persons uncontrollable impulses).

Time. Another phenomenon in consultancy and psychology is that many problems or complaints and their accompanying consequences may fade away or at least will become bearable through time. Of course, the question remains how much time should be practiced and to what extent the causes underlying the problems and complaints will be solved or that it is merely a dangerous process of habituation, like the boiled frog metaphor. Also, the other way around, early intervening is important, given that prevention in both consultancy and psychology has proven to be much more effective and efficient than curative action.

Organisations. In organisations, a lot of mechanisms may be in place to cope with events, among which strategies, structures, systems and processes as well as cultures, leaderships, capabilities and behaviors. However, an organisation may face triggers (for instance dropping demand, disruptive technologies or bulky legislations) that are so highly intense and/or so highly frequent, that the available assortment of mechanisms is not adequate any more to cope with these events. In those cases, an organisation will face a "crisis "or "breakdown". One of the fundamental characteristics of such a crisis is that doing or adding "more of the same" will not solve the crisis, however, nevertheless this often seems to be the default-reaction. Generally, adding more of the same will only spoil scarce resources and will cause exhaustion, while adding complexity in fighting and compensating the symptoms, instead of taking away the underlying causes, including their maintaining processes.

Persons. As in organisations, also in relation to persons, it may be the case that the frequency and/or the intensity of certain trigger events (for instance financial problems, relation problems, work problems, or other life disruptions), called "stressors", may be perceived as threatening or fearful to an extent that a crisis or "mental breakdown" may occur. As in organisations, everyone has a range of more or less effective protection mechanisms available to prevent or mitigate mental breakdowns. However, the sum of all stressors versus the so called "bearing capacity" of a person, determines situationally how much stress a certain person is able to handle before the threshold from a mental breakdown is exceeded. This may well be compared to the origination of a breakdown in organisations, and may even be more narrowly related to organisations, if and when leaders are not able anymore to function adequately, because of stress.

Protection. As explained, both organisations and persons have an assortment of more or less conscious, as well as more or less effective protection mechanisms available. According to my opinion, important archetypes of these protection mechanisms are support systems as well as appraisal styles and coping strategies. Support systems include the help from colleagues and professionals as well as family and friends. Appraisal styles are for instance the perceived locus of control and the way of attributing events (own contribution versus environmentally). Coping strategies have lots of examples and cover among others, primitive and sophisticated defence mechanisms (Freud 2013) such as denials and projections. Compensating behaviors may also be 
protective, and may take the form of addictions, for instance unceasingly introducing fashionable templates in relation to organisations and drinking or drugs in relation to persons. If these mechanisms are not able to protect sufficiently, a breakdown in a person or an organisation may occur. Also, the exclusive fixation on a limited set of protection mechanisms may not be sufficient to handle the variety of trigger events, causing a breakdown.

Personalities. As in psychology also in consultancy, 'personality disorders' may be observable, for instance in the form of deviant leadership characteristics. From the perspective of consultancy, these personality disorders should at least be recognised and taken into consideration. This is also because the tails of the normal distributions of personalities may cause severe problems in organisations, apart from problems for the persons themselves (Kets de Vries 1984). In these cases, deviant leadership styles or behaviors may form the root cause underlying the more superficial organisational problems and complaints. A personality disorder may be defined as 'an enduring pattern of inner experience and behavior that deviates markedly from the expectations of the individual's culture, is pervasive and inflexible, has an onset in adolescence or early adulthood, is stable over time and leads to distress or impairment' (DSM 2013). Personality disorders may be roughly summarised in three basic categories, each with its own pathology. These categories of personality disorders are in my terms "overly suspicious", "overly emotional", and "overly anxious".

Psychiatric. "psychiatric disorders" certainly should not be in scope of consultancy. That being said, all kinds of manifestations of characteristics stemming from psychiatric disorders may also be observed in consultancy. Examples of psychiatric disorders may be rich sets of symptoms, for instance around depressions (with attributes as indecisiveness and hopelessness) and mania (with attributes as grandiosity and risktaking) as well as obsessions and compulsions. These psychiatric disorders may also underly organisational problems, especially in relation to top management.

Doctrines. In both consultancy and psychology, one of the ongoing questions is to what extent a comprehensive prestructuring and protocoling of "all problems" around diagnosing and "all remedies" around constructing adds value. Apart from the value, according to my opinion, such enumerations are not even feasible, also given the many situational differences in the current situation as well as the need for tailored and feasible solutions. After all, strongly prestructured approaches may result in "ticking-thebox" exercises, with the risk of more attention being paid to the mechanistics and the form than to the organics and the substance. Also, a strong prestructuring of remedies may promote the use of templates, with inherent biases to one-size-fits-all "solutions". Furthermore, templates may even give rise to a specific risk, if and when these templates are used by less seasoned professionals, with the function of substituting experience by procedures.

Complications. For problems and complaints in organisations and persons often many alternative explanations are both available and plausible. As mentioned, one of the most important activities in both consultancy and psychology, is to adequately relate superficial symptoms to underlying root causes, in order to identify appropriate and sustainable changes. Apart from all other distortions that may occur, professionals in consultancy as well as in psychology may misjudge associations for causations and 
thus reach the wrong conclusions about the cause(s) underlying the problems. Even more important, if and when the ecosystem, in which organisations and persons exist and interact, are not sufficiently taken into account, only parts may change, leaving the whole unchanged.

Norms. Likewise, in consultancy and psychology, assessing and weighting symptoms, including the distance to the desired or required state, is highly dependent on for instance the way of perceiving situations and events plus the models and methods used by the professionals involved. Ideally, a clear and well demarcated set of norms should be available, against which the current and the proposed situation can be assessed. This has to be the case in consultancy and psychology in order to work "evidence based", which among others implies that the work is reproducible over professionals. After all, organisational or mental breakdowns are nothing more than a discrepancy between a certain norm versus an observed composition or functioning.

Focus. In consultancy as in psychology, a tendency may be signalled to focus on the more "hard" phenomena, may be because these often are more visible or because they are perceived to be "safer" to handle. In psychology, such an approach would translate itself into the medicalisation of all problems and complaints. In consultancy, this may translate itself in a single-sided hard focus on for instance digitalisation, big data and artificial intelligence. This single-sidedness may express itself in the risk of only more plans and paper as well as disappointments, without cashing in the expected effects. Also, suppressing the soft-side, may result in not sufficiently taking away the underlying root causes and the maintaining processes, often having behavioral components. This also implies that the root cause(s) will still exist, doing their destructive work under the surface.

Resistance. Also, problems and complaints in organisations and persons often stem from the pressures put on them, for instance in organisations higher returns or higher quality and in persons career demands or life balance. Within organisations, problems may come from obsolete systems and structures, while in both organisations and persons problems may come from obsolete behaviors and competencies. In all cases, early assessments and interventions are valuable, in order to prevent unnecessary escalations, resulting in breakdowns. However, in organisations and in persons, a range of resistance mechanisms is in place, hindering early assessments and interventions in more or less subtle ways. This also underpins the relations between consultancy as well as psychology in terms of coping with these forms of collective or individual resistance.

Biases. Numerous cognitive biases exist, distorting the creation of fair models of situations and events. From that point of view, these biases may hinder both adequate diagnoses and treatments of organisational and personal problems, among others given the more or less unconscious mental shortcuts towards judgements and decisions. Some examples of the hundreds of these biases are anchoring biases (tendency to rely solely on a limited set of characteristics), attribution biases (tendency to claim successes and to wave away involvement in failures), authority biases (tendency to overvalue the opinions of specialists), belief biases (tendency to weight arguments in the light of the believability of their conclusion), completeness biases (tendency to seek for additional data even when not helpful anymore), confirmation biases (tendency to search for and interpret data in line with preconceptions, while rejecting conflicting 
data), courtesy biases (tendency to provide socially accepted opinions instead of true convictions), egocentric biases (tendency to reconstruct parts of the past in a selfserving manner), hostile biases (tendency to interpret behavior as having harmful intents), result biases (tendency to judge decisions on the product instead of the quality of the process), stereotyping biases (tendency to have judgements or opinions about people of situations without accurate information) etc.

\section{Final Comments}

One of the challenges in progressing a profession, is to initiate and continue productive debates around the more fundamental and thought provoking questions, without the competent use of our own defence mechanisms. In line with this contribution, some of these questions for consultancy may be amongst others the following.

- To what extent are pro-active assessments and early interventions (possibly preventing crises) offered?

- To what extent are total ecosystems sufficiently taken into scope in all phases of a change process?

- To what extent are hard and soft phenomena situationally in balance in all phases of a change process?

- To what extent are product versus process emphasis situationally considered in all phases of a change process?

- To what extent are indicators available and used about the normality versus the abnormally of situations?

- To what extent are clear norms available and applied in consultancy in order to work evidence-based?

- To what extent are problems and complaints analysed in unnecessarily overly detailed ways?

- To what extent are maintaining processes underlying problems recognised and sufficiently taken into account?

- To what extent are templates misused as substitute for seniority in diagnosing, constructing and changing?

- To what extent are forms of resistance identified as well as handled and used in a productive way?

- To what extent are key persons situationally more productive points of attack as compared to organisations? 
- To what extent are deviant leadership styles the root cause underlying superficial organisational problems?

- To what extent are personality disorders taken into account in consultancy and should that be the case anyway?

- To what extent are cognitive biases and other distortions identified as well as sufficiently mitigated?

- To what extent are psychological accomplishments (sufficiently) integrated in the education of consultants? 


\section{References}

DSM 2013 - diagnostic and statistical manual of mental disorders, 2013, ISBN 9780890425558

Freud 2013 - das Ich und die Abwehrmechanismen, reprint, 2013, ISBN 9785518929128

Kets de Vries 1984 - the neurotic organisation, 1984, ISBN 0875896065. 